Original Paper http://ajol.info/index.php/ijbcs http://indexmedicus.afro.who.int

\title{
Enquête ethnobotanique sur les plantes utilisées traditionnellement au Niger dans la lutte contre les moustiques vecteurs des maladies parasitaires
}

\author{
Issoufou YOLIDJE ${ }^{1 *}$, Djibo ALFA KEITA ${ }^{1}$, Idrissa MOUSSA ${ }^{1}$, Abdoulaye TOUMANE $^{2}$, \\ Sahabi BAKASSO ${ }^{1}$, Karim SALEY ${ }^{3}$, Tilman MUCH $^{4}$, Jean-Luc PIRAT ${ }^{5}$ et \\ Jean Maurille OUAMBA ${ }^{6}$ \\ ${ }^{1}$ Laboratoire des Substances Naturelles, Faculté des Sciences et Techniques, Université Abdou Moumouni, BP \\ 10667, Niamey, Niger. \\ ${ }^{2}$ Laboratoire Garba Mounkaila, Département de Biologie, Faculté des Sciences et Techniques, Université \\ Abdou Moumouni. BP 10667, Niamey, Niger. \\ ${ }^{3}$ Université Dan Dicko Dan koulodo de Maradi, Niger. \\ ${ }^{4}$ Université de Bayreuth, Allemagne. \\ ${ }^{5}$ Laboratoire de Chimie Organique (UMR 5076 du CNRS), Ecole Nationale Supérieure de Chimie de \\ Montpellier, 8 Rue de l'Ecole Normale, 34296 Montpellier Cedex 5, France. \\ ${ }^{6}$ Université Marien NGouabi, Congo Brazzaville. \\ *Auteur correspondant ; E-mail: yolidjeissouf@yahoo.com; Tel : +227 98395897
}

\section{RESUME}

Le paludisme apparaît comme la plus vieille et la plus meurtrière des maladies tropicales. A travers le monde, de nombreux peuples ont utilisé traditionnellement des plantes pour lutter contre cette maladie. Pour contribuer à la lutte préventive contre le paludisme, une enquête ethnobotanique a été menée à Niamey au près des 40 tradipraticiens afin inventorier les plantes spontanées présumées avoir des activités insecticides. Il ressort des résultats de cette enquête, une nette prédominance des hommes dans l'exercice de la médecine traditionnelle au Niger. En effet, sur les 40 tradipraticiens enquêtés, 36 sont des hommes contre seulement 4 femmes. L'enquête a permis de recenser 28 espèces végétales potentiellement insecticides. Ces plantes sont reparties dans plusieurs familles, celles les plus représentatives sont les Papilionaceae $(21,4 \%)$, les Lamiaceae $(7,1 \%)$, les Euphorbiaceae $(7,1 \%)$, les Combretaceae $(7,1 \%)$ et les Capparidaceae $(7,1 \%)$. Les plantes fréquemment citées sont: Azadirachta indica (21\%), Striga hermontheca $(13,9 \%)$ et Hyptis spicigera $(10,0 \%)$. Les parties des plantes les plus utilisées sont principalement les feuilles $(53,3 \%)$ et les tiges feuillées $(33,3 \%)$, avec comme mode principal d'utilisation la fumigation $(82,0 \%)$. Ces résultats pourraient servir dans le domaine de la recherche des nouveaux biopesticides. L'investigation sera étendue à d'autres régions du pays afin de rassembler le maximum d'espèces végétales antipaludiques.

(C) 2020 International Formulae Group. All rights reserved.

Mots clés: Enquête ethnobotanique, tradipraticiens, plantes insecticides, moustiques, paludisme.

\section{Ethnobotanical survey on plants traditionally used in Niger in the fight against mosquitoes vectors of parasitic diseases}

\section{ABSTRACT}

Malaria appears to be the oldest and deadliest tropical disease. Throughout the world, many people have traditionally used plants to fight this disease. To contribute to the preventive fight against malaria, an ethnobotanical survey has been carried out in Niamey with traditional practitioners to inventory spontaneous 
plants presumed to have insecticidal activities. The survey shows a predominance of men in the sector; thus, of the 40 traditional healers surveyed, we have 36 men and 4 women. The survey identified 28 potentially insecticidal plant species. These plants are distributed in several families, the most representative are Papilionaceae (21.4\%), Lamiaceae (7.1\%), Euphorbiaceae (7.1\%), Combretaceae $(7.1 \%)$ and Capparidaceae (7.1\%). The most frequently mentioned plants are: Azadirachta indica (21\%), Striga hermontheca (13.9\%) and Hyptis spicigera $(10.0 \%)$. The most commonly used plant parts are mainly leaves $(53.3 \%)$ and leafy stems $(33.3 \%)$, with fumigation as the main mode of use (82.0\%). These results could be used in the field of research of new biopesticides. The investigation will be extended to other regions of the country to gather the maximum number of plant antimalarial species.

(C) 2020 International Formulae Group. All rights reserved.

Keywords: Ethnobotanical survey, traditional healers, medicinal plants, mosquitoes, malaria.

\section{INTRODUCTION}

Le continent africain dispose d'une diversité importante de plantes médicinales (Dibong et al., 2011). Aussi, plus de 80\% de la population africaine fait recours à la médecine traditionnelle en utilisant des plantes médicinales pour résoudre leurs problèmes de santé primaire (WHO, 2002). L'ethnobotanique recouvre le champ des interrelations entre le monde végétal, le monde des remèdes et celui des cultures et s'inspire de l'ethnologie dans ses méthodes d'investigations (Rivière et al., 2005). Par ailleurs, la place importante qu'occupent les plantes médicinales en tant que substrat le plus important des recettes de la médecine et de la pharmacopée traditionnelles n'est plus à démontrer. La population locale, par son expérience et par les savoirs empiriques transmis par les générations précédentes, reconnait les plantes médicinales dans leur milieu naturel par différents aspects (odeur, couleur des fleurs, forme des feuilles, localisation, saison, etc.) (Riviere et al., 2013). Ces dernières constituent une importante source naturelle de biomolécules susceptibles de receler d'innombrables propriétés pharmacologiques à même de guérir ou de circonscrire un grand nombre de pathologies humaines telle que le paludisme qui est l'une des maladies qui cause le plus de décès dans le monde, particulièrement en Afrique et au Niger. Dans le cadre de la recherche de nouvelles molécules naturelles à effet insecticide dans la lutte contre les moustiques vecteurs du paludisme, nous avons mené dans la présente étude une enquête ethnobotanique sur les plantes utilisées au Niger pour chasser les moustiques.

\section{MATERIEL ET METHODES}

\section{Présentation de la zone d'Etude}

La Communauté urbaine de Niamey créée par la Loi n 98-32 du 14 Septembre 1998 est une des huit régions du Niger. Située entre $2^{\circ} 01^{\prime} 43^{\prime \prime}$ et $2^{\circ} 14^{\prime} 05^{\prime \prime}$ de longitude Est et $13^{\circ} 25^{\prime} 45^{\prime \prime}$ ' et $13^{\circ} 36^{\prime} 16^{\prime \prime}$ de latitude Nord. Niamey couvre une superficie de 239,263 km². Avec une altitude variant de 180 à $250 \mathrm{~m}$. La ville est coupée en deux par le fleuve Niger (Figure 1). La rive gauche abrite quatre communes (1 à 4), est un plateau qui surplombe le fleuve à une altitude moyenne de $220 \mathrm{~m}$. Quant à la rive gauche (Commune 5) c'est une plaine alluviale d'une altitude moyenne de 185 $\mathrm{m}$. Capitale politique et siège de la plupart des unités industrielles et économiques, Niamey a connu des croissances démographiques et spatiale rapides au cours des quarante dernières années. La population qui n'était que de 33816 habitants en 1960, puis de 707951 en 2001, est estimée à 1033295 en 2008, soit une multiplication par 30,5 en moins de 50 ans. Aux mêmes moments la superficie s'est accrue passant de 2347 ha en 1972 à 4400 en 1977 et à près de 11000 ha au début des années 2000 (Issaka et Badariot, 2013). 


\section{Enquête ethnobotanique}

L'enquête ethnobotanique a été réalisée à l'aide d'un questionnaire pré-élaboré en utilisant un entretien semi-directif. Elle a été conduite dans 10 marchés de vente des plantes médicinales de la région de Niamey de juin à juillet 2014. Il s'agit des marchés : Aéroport, Boukoki, Gamkalé, Harobanda, Talladjé, Katako, Koirategui, Lazaret, Saga et Wadata (Figure 1). Les questions ont été posées dans la langue de l'enquêté (Haoussa, Zarma ou Peul). Au total 40 tradipraticiens ont été interrogés. L'échantillonnage était de type aléatoire dans les marchés visités. Le renseignement a porté sur les plantes utilisées traditionnellement comme insecticides contre les moustiques. La fiche d'enquête utilisée portait des informations concernant le profil de la personne ressource (âge, sexe, niveau d'étude, etc.) et les noms locaux des plantes, la partie de la plante utilisée, les modes de préparation et d'utilisation de ces plantes. Les noms scientifiques des plantes recensées lors de l'enquête et récoltées, ont été déterminés au Laboratoire Garba Mounkaila du département de Biologie de l'Université Abdou Moumouni de Niamey, par comparaison aux échantillons disponibles dans leur herbier. La liste des espèces végétales recensées au cours de l'enquête ethnobotanique a également été comparée à celles du rapport de mission du ministère de la santé publique sur les plantes médicinales du Niger (MSP, 1985) et de la flore du Sénégal (Jacques, 2008). Les données enregistrées sur les fiches d'enquête ont été ensuite saisies et analysées avec le logiciel Microsoft Excel 2010. La fréquence relative de citation ou (FRC) a été calculée en utilisant l'Équation (1) :

FRC

$=\frac{\text { Frequence de citation par plante }}{\text { Nombre total de refences }} \times 100$

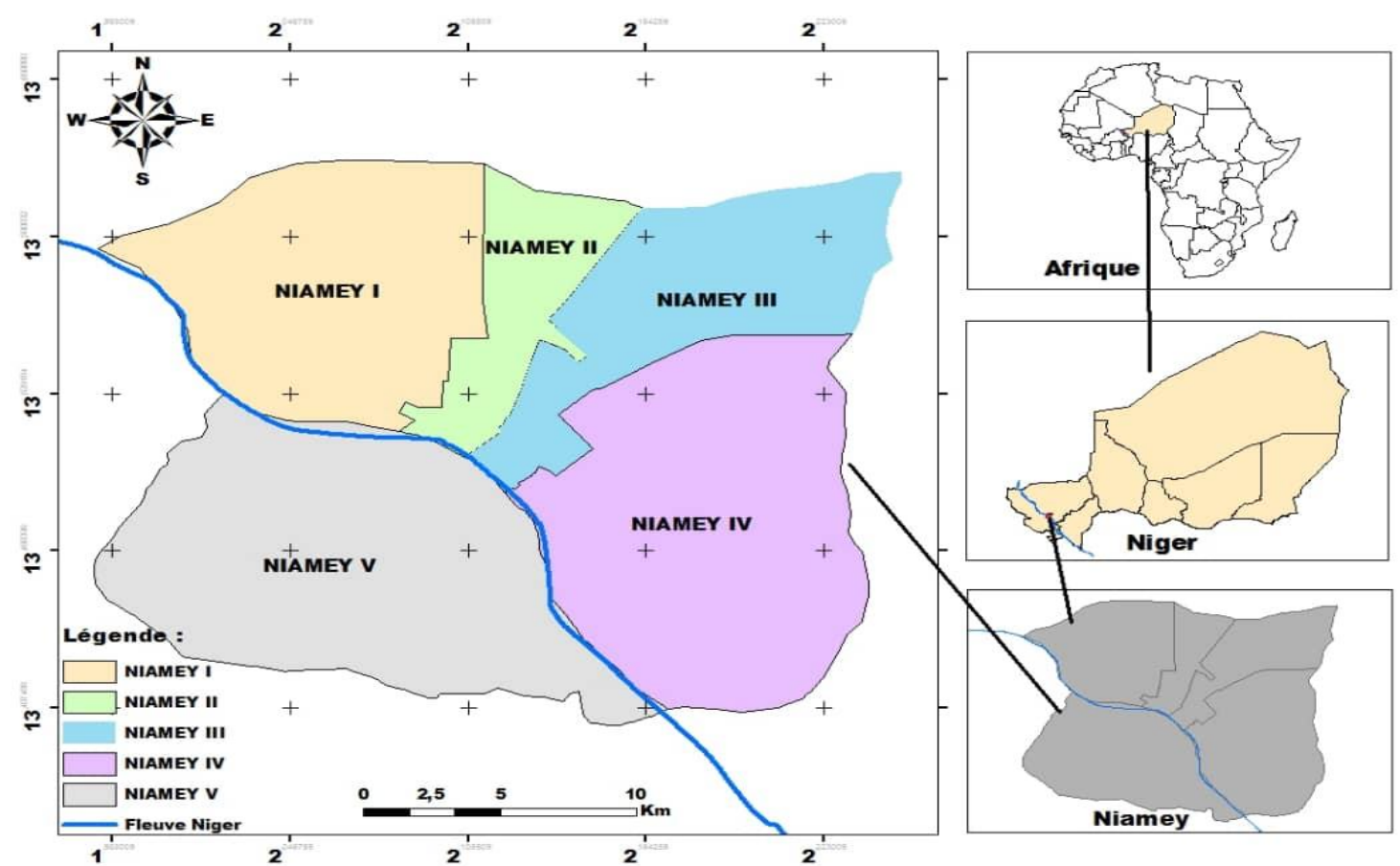

Figure 1 : Présentation de la zone d'étude. 


\section{RESULTATS}

Les résultats de l'enquête ethnobotanique montrent une prédominance des hommes dans l'exercice de la médecine traditionnelle au Niger. En effet, sur les 40 tradipraticiens enquêtés, 36 sont des hommes contre 4 femmes seulement (Figure 2). On constate dans la présente étude que la langue de communication de $80 \%$ des enquêtés est le Haoussa contre $15 \%$ qui communiquent en Zarma et 3\% en Peul (Figure 3). Cela suppose que le métier de tradipraticien persiste aujourd'hui encore fortement au Niger et principalement dans cette communauté.

On note également que l'âge moyen des tradipraticiens rencontrés est autour de quarante-huit ans (48 ans), le plus jeune a 27 ans et le plus âgé a 80 ans. Les sujets d'au moins soixante $(60$ ans) représentent plus du tiers de la population enquêtée (Figure 4), ceci pourrait justifier une importante expérience des tradipraticiens dans la pharmacopée traditionnelle au Niger. Les résultats montrent aussi que, seul $42 \%$ des tradipraticiens

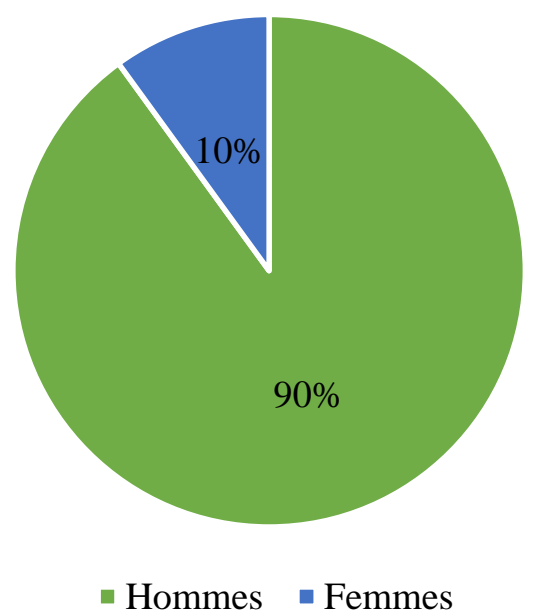

Figure 2: Répartition des tradipraticiens selon le genre. enquêtés ont été scolarisés contre 58\% non scolarisés (Figure 5).

A la suite de l'enquête ethnobotanique, vingt-huit (28) espèces végétales utilisées contre les moustiques ont été recensées. Ces espèces végétales sont regroupées en vingtcinq (25) genres et appartiennent à dix-huit (18) familles (Tableau 1). Les familles les plus représentatives sont les Papilionaceae $(21,4 \%)$, les Lamiaceae (7,1\%), les Euphorbiaceae $(7,1 \%)$, les Combretaceae $(7,1 \%)$ et les Capparidaceae $(7,1 \%)$. Les résultats de l'enquête ethnobotanique montrent que les espèces végétales les plus utilisées pour lutter contre les moustiques au Niger sont: Azadirachta indica (21,1\%), Striga hermontica (14\%) et Hyptis spicigera (10,5\%) (Figure 6). Les parties des plantes les plus utilisées sont principalement les feuilles $(53,3 \%)$ et les tiges feuillées $(33,3 \%)$, la plante entière et les fruits ne sont pas utilisées (Figure 7). Elles sont suivies par les écorces, les racines et les graines. Le mode d'utilisation est principalement la fumigation $(82,0 \%)$.

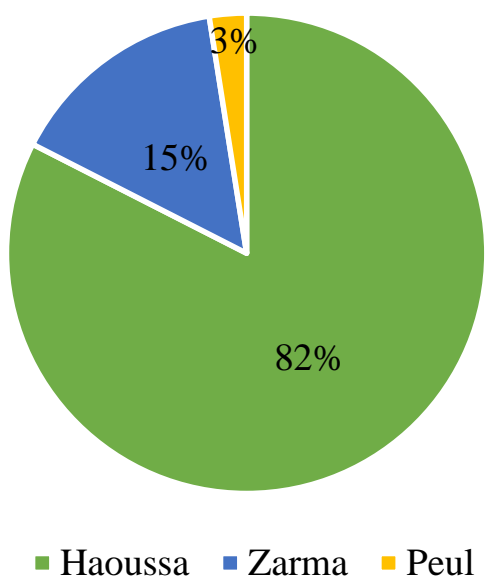

Figure 3: Répartition des tradipraticiens enquêtés selon la langue parlée. 


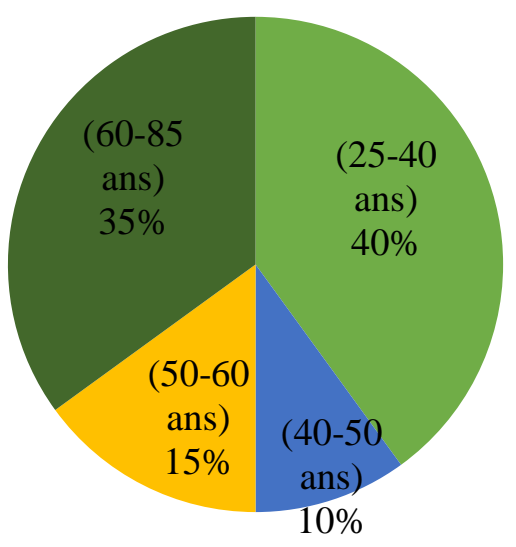

Figure 4: Répartition des tradipraticiens selon l'âge.

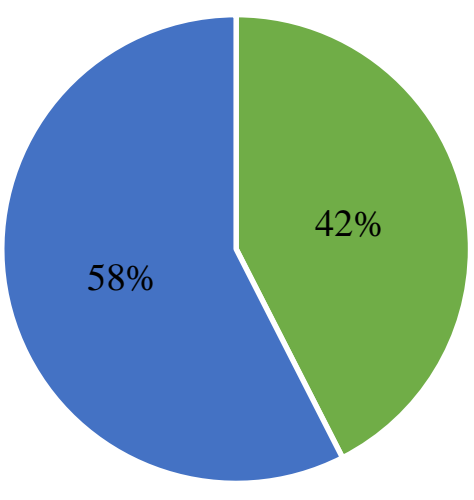

- Scolarisés - Non scolarisés

Figure 5: Répartition des tradipraticiens selon le niveau d'étude.

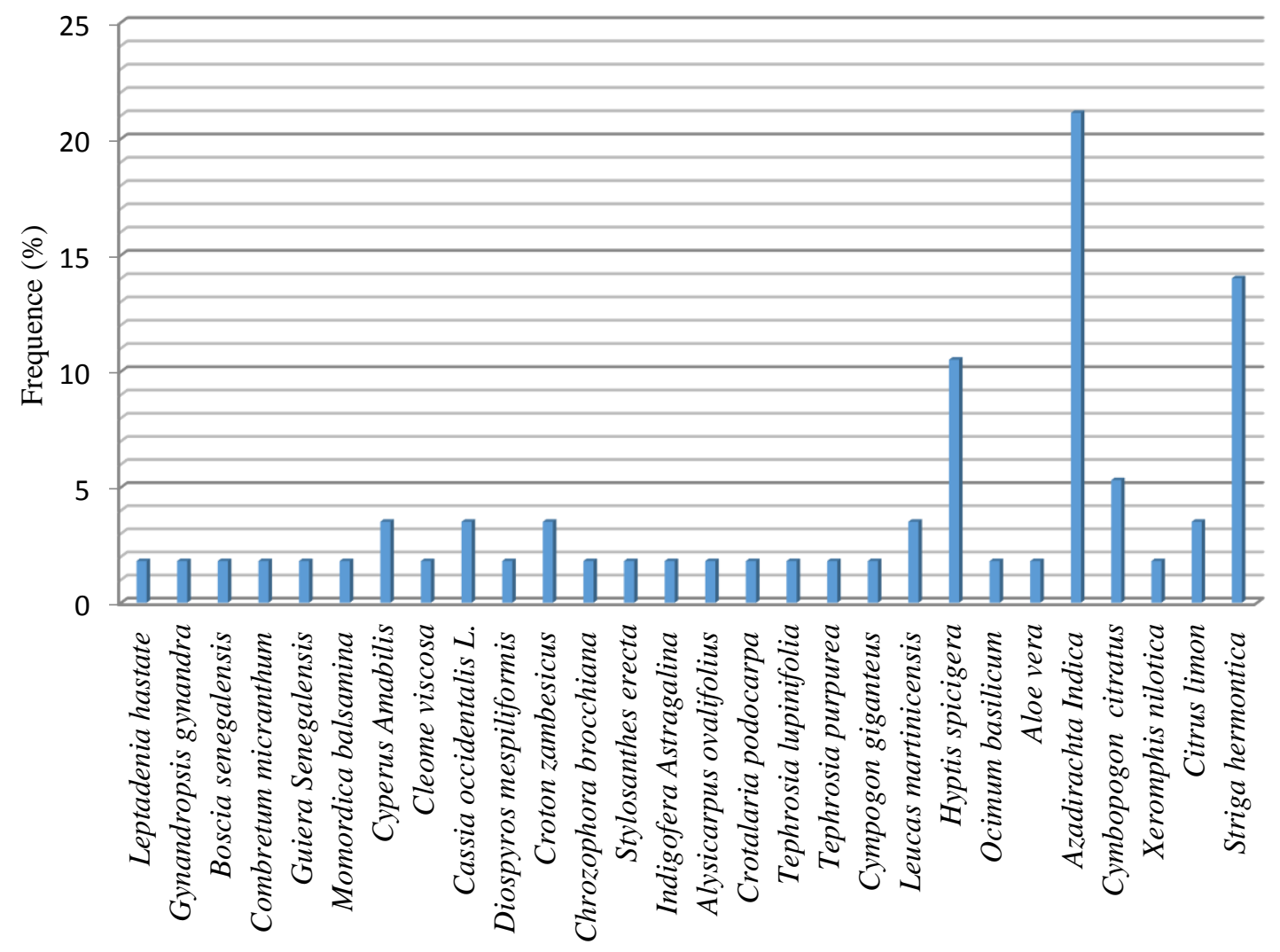

Figure 6 : Espèces végétales utilisées dans la lutte contre les moustiques. 
Tableau 1: Espèces végétales recensées lors de l'enquête ethnobotanique.

\begin{tabular}{|c|c|c|c|c|c|}
\hline Noms scientifiques & $\begin{array}{l}\text { Familles } \\
\text { botaniques }\end{array}$ & Noms vernaculaires & Organes utilisés & Mode d'utilisation & FRC \\
\hline Leptadenia hastate (Pers.) Decne & Asclepediaceae & H : Yadya Z: Hanam P : Yadyahul & Tiges feuillées & Fumigation (Insectifuge) & 1,8 \\
\hline Gynandropsis gynandra & Capparidaceae & H: Gasaya Z: Houbey P: Fubey & Feuilles & Fumigation (Insectifuge) & 1,8 \\
\hline $\begin{array}{l}\text { Boscia senegalensis (Pers.) Lam. Ex } \\
\text { Poir }\end{array}$ & Capparidaceae & H: Anza Z: Orha P : Gigilé & Feuilles & Fumigation (Insectifuge) & 1,8 \\
\hline Combretum micranthum G.Don. & Combretaceae & H : Géza Z : Kubu P : Talli & Feuilles & Fumigation (Insectifuge) & 1,8 \\
\hline Guiera Senegalensis J.G. Gmel. & Combretaceae & H : Sabra Z : Sabara P : Gélokhi & Feuilles & Fumigation (Insectifuge) & 1,8 \\
\hline Momordica balsamina $L$. & Cucurbitaceae & H : Garahuni Z :BadomaP:Jelanfettohi & Feuilles & Fumigation (Insectifuge) & 1,8 \\
\hline Cyperus AmabilisVahl. & Cyperaceae & H : Jiji Z : Dugu P : Uhguldi & Racines & Fumigation (Insectifuge) & 3,5 \\
\hline Cleome viscosa & Cleomaceae & $\mathrm{H}$ : gashya mahalba $\mathrm{Z}$ : jéri lanpti & Tiges feuillées & Fumigation (Insectifuge) & 1,8 \\
\hline Cassia occidentalis $L$. & Caesalpinaceae & $\mathrm{H}$ : Kinkilba Z : Sangasanga P : Kashiukashiuki & Feuilles & $\begin{array}{l}\text { Décoction } \\
\text { (antiplasmodiale) }\end{array}$ & 3,5 \\
\hline $\begin{array}{l}\text { Diospyros mespiliformis Hochst. ex. A. } \\
\text { DC }\end{array}$ & Ebenaceae & H : Kanya Z : Tokey P : Nelbi & Feuilles & Fumigation (Insectifuge) & 1,8 \\
\hline Croton zambesicus Muell & Euphorbiaceae & H : Koriba Z : Tondibonheyni & Tiges feuillées & Fumigation (Insectifuge) & 3,5 \\
\hline Chrozophora brocchiana Vis. & Euphorbiaceae & H : Damagui Z : Dorey P : Dusur & Feuilles & Fumigation (Insectifuge) & 1,8 \\
\hline Stylosanthes erectaP. Beauv. & Papilionaceae & $\mathrm{H}:$ Tsirahoko & Tiges feuillées & Fumigation (Insectifuge) & 1,8 \\
\hline Indigofera Astragalina DC. & Papilionaceae & H : Hakorindoki Z : Sujikuku P: Irimadjogohi & Feuilles & Fumigation (Insectifuge) & 1,8 \\
\hline $\begin{array}{l}\text { Alysicarpus ovalifolius (Schum. Et } \\
\text { Thonn.) }\end{array}$ & Papilionaceae & H : Gadagy Z : Gadagy $\mathrm{P}$ : Gadagyl & Tiges feuillées & Fumigation (Insectifuge) & 1,8 \\
\hline
\end{tabular}


I. YOLIDJE et al. / Int. J. Biol. Chem. Sci. 14(2): 570-579, 2020

\begin{tabular}{|c|c|c|c|c|c|}
\hline Crotalaria podocarpa DC. & Papilionaceae & $\mathrm{H}$ : Gonda $\mathrm{Z}$ : Yomovano P : Goddaro & Feuilles & Fumigation (Insectifuge) & 1,8 \\
\hline Tephrosia lupinifoliaDC. & Papilionaceae & $\mathrm{H}$ : Gudjyalkasa $\mathrm{Z}$ : Ganda damsi P : Kolhitati & Tiges feuillées & Fumigation (Insectifuge) & 1,8 \\
\hline Tephrosia purpurea(L.) Pers. & Papilionaceae & $\mathrm{H}$ : Massa Z : Massa P : Masahy & Tiges feuillées & Fumigation (Insectifuge) & 1,8 \\
\hline Cympogon giganteus Chiov. & Gramineae & H : Tsabré Z : Gosofaryé P : Gadjiali & Tiges feuillées & Fumigation (Insectifuge) & 1,8 \\
\hline Leucas martinicensis $\mathrm{L}$ & Lamiaceae & $\begin{array}{l}\text { H : Sarakwarsabro Z : Sabroanzurey P : lulu- } \\
\text { heyné }\end{array}$ & Tiges feuillées & Fumigation (Insectifuge) & 3,5 \\
\hline Hyptis spicigera Lam. & Lamiaceae & $\mathrm{H}:$ Wari Z : KareyWakondo & Tiges feuillées & Fumigation (Insectifuge) & 10,5 \\
\hline Ocimum basilicum $L$. & Liliaceae & $\mathrm{H}$ : Kafi amaria kamchi Z : Korogabou & Tiges feuillées & Fumigation (Insectifuge) & 1,8 \\
\hline Aloe vera & Liliaceae & $\mathrm{H}:$ Aloe vera, $\mathrm{Z}:$ Aloe vera & Partie aérienne & Fumigation (Insectifuge) & 1,8 \\
\hline Azadirachta Indica A. Juss. & Meliaceae & $\mathrm{H}$ : Dogonyaro $\mathrm{Z}$ : Turiforta $\mathrm{P}:$ Miliihi & Feuilles & Fumigation (Insectifuge) & 21 \\
\hline Cymbopogon citratus(DC.) Stapf. & Poaceae & $\mathrm{H}$ : Citronelle $\mathrm{Z}$ : Citronelle & Feuilles & Fumigation (Insectifuge) & 5,3 \\
\hline Xeromphis nilotica(Stapf.) Keay. & Rubiaceae & $\mathrm{H}$ : Chibra Z : Lilo & Racines & Fumigation (Insectifuge) & 1,8 \\
\hline Citrus sinensis & Rutaceae & $\mathrm{H}$ : uwa Lemou $\mathrm{Z}$ : Lemou gna & Péricarpesdes fruits & Fumigation (Insectifuge) & 3,5 \\
\hline Striga hermontica (Del.) Benth. & Scrophulariaceae & H : Gawgaye Z : Malli P :Ngudugi & Tiges feuillées & Fumigation (Insectifuge) & 14 \\
\hline
\end{tabular}




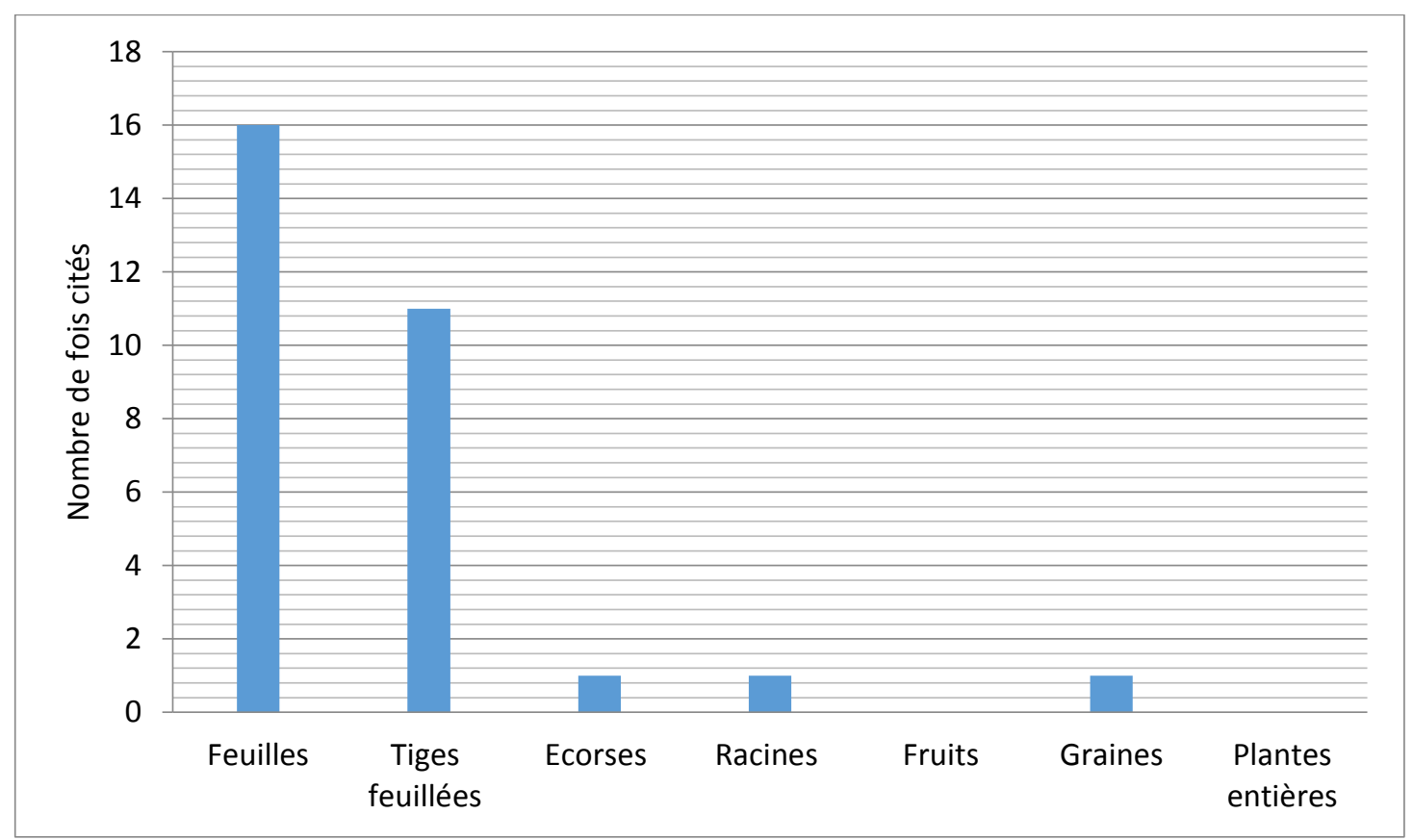

Figure 7: Organes des espèces végétales utilisées dans la lutte contre les moustiques.

\section{DISCUSSION}

Les résultats de l'enquête ethnobotanique montrent une prédominance des hommes dans l'exercice de la médecine traditionnelle au Niger. Cela infirme les résultats des études réalisées au Mali par Dénou et al. (2017) qui avaient trouvé que les femmes pratiquaient majoritairement cette tâche $(70,8 \%)$. L'enquête ethnobotanique a montré que les espèces végétales les plus utilisées pour lutter contre les moustiques au Niger sont: Azadirachta indica, Striga hermontica et Hyptis spicigera. Des résultats similaires ont été rapportés par plusieurs auteurs. Au Cameroun, Hyptis spicigera et Azadirachta indica ont été rapportés comme espèces végétales les plus utilisées pour lutter contre les moustiques (Innocent et al., 2010). A la différence, ces auteurs n'ont pas rapporté l'utilisation de Striga hermontica. Au Togo, Cymbopogon citratus (DC.) Stapf, Azadirachta indica L. et Hyptis sauveolens Poit sont rapportées comme espèces végétales les plus utilisés dans la lutte antipaludique (Koudouvo et al. ; 2011). Hyptis sauveolens n'a été citée dans la présente étude. En République Démocratique du Congo, 46 plantes antipaludiques ont été recensées parmi lesquelles, Azadiratcha indica et Cymbopognon citratus sont les plus citées (Kasali et al., 2014). En
Côte d'Ivoire, il a été rapporté l'utilisation de 57 espèces végétales antipaludiques parmi lesquelles Azadirachta indica et Cymbopogon citratus sont les plus utilisées (N'GUESSAN et al., 2009). Cependant, des résultats différents ont été rapportés au Mali, ces auteurs ont rapportés Mitragyna inermis et Anogeissus leiocarpus comme espèces végétales antipaludiques les plus utilisées

De plus, parmi les 28 espèces végétales recensées dans la présente étude les activités insecticides des 12 plantes ont été rapportés. Il s'agit de: Azadirachta indica (Seye et al.; 2006. ; Saotoing et al., 2014); Striga hermonthica (Abdullahi et al., 2011); Cleome viscosa (Bansal et al., 2014); Cymbopogon citratus (Karunamoorthi et Ilango, 2010); Citrus lemon (Khan et al., 2015); Momordica balsamina (Khan et al., 2015); Ocimum basilicum (Belong et al.; 2013); Ocimum americanum (Belong et al., 2013); Khaya senegalensis (Saotoing et al., 2014); Cassia occidentalis (Abirami et al., 2011) et B. senegalensis (Gueye et al., 2011). L'utilisation principale des feuilles dans la pharmacopée traditionnelle est rapportée par plusieurs auteurs (Lawaly et al., 2017 ; N'guessan et al., 2009 ; Denou et al ., 2017). 


\section{Conclusion}

L'enquête ethnopharmacologique réalisée dans la ville de Niamey a permis de recenser 28 espèces végétales potentiellement insecticides. Ces espèces sont reparties dans plusieurs familles, celles les plus représentatives sont les Papilionaceae et les Lamiaceae. Les plantes fréquemment citées sont : Azadirachta indica, Striga hermontheca et Hyptis spicigera. Les parties des plantes les plus utilisées sont principalement les feuilles et les tiges feuillées, avec comme mode principal d'utilisation la fumigation $(82,0 \%)$. Il est indispensable d'évalué l'efficacité des plantes recensées par des études expérimentales appropriées telle que l'étude de l'activité larvicide des huiles essentielles et les extraits bruts pour que leur usage soit davantage fondé scientifiquement.

\section{CONFLITS D'INTÉRÊTS}

Tous les auteurs déclarent qu'il n'y a aucun conflit d'intérêts pour la publication de cet article.

\section{CONTRIBUTIONS DES AUTEURS}

IY, IM, SB ont participé à l'élaboration du protocole, supervisé sa mise application et la rédaction de l'article. KS a été utile pour l'identification des plantes et a participé à l'écriture et à l'amélioration du manuscrit. AT, TM, JLP, JMO ont apporté des commentaires sur le document. Enfin, DAK a proposé le thème, a encadré le travail et a finalisé la rédaction du manuscrit.

\section{REFERENCES}

Abdullahi K, Abubakar MG, Umar RA, Gwarzo MS, Muhammad M, Ibrahim HM. 2011. Studies on the larvicidal efficacy of aqueous extracts of Striga hermonthica (Delile) Benth and Mitracarpus scaber (Zucc) on Culex quinquefasciatus (Culicidae) mosquito larvae. Journal of Medicinal Plants Research, 5(21):5321-5323.

Abirami D, Murugan K. 2011. HPTLC quantification of flavonoids, larvicidal and smoke repellent activities of Cassia occidentalis L. (Caesalpiniaceae) against malarial vectore Anopheles stephensi Lis (Diptera: Culicidae. Journal of Phytology, 3(2): 60-72.

Bansal SK, Singh V, Sharma SS. 2014. Larvicidal potential of wild mustard (Cleome viscosa) and gokhru (Tribulus terrestris) against mosquito vectors in the semi-arid region of Western Rajasthan. Journal of Environmental Biology, 35(2): 327-332.

Belong P, Ntonga PA, Bakwo EM, Dadji GAF, Tamesse JL. 2013. Chemical composition and residue activities of Ocimum canum Sims and Ocimum basilicum L essential oils on adult female Anopheles funestus ss. Journal of Animal \&Plant Sciences, 19(1): 2854-2863.

Dibong SD, Emmanue MML, Alfred NBJ, France KM. 2011. Ethnobotanique et phytomédecine des plantes médicinales de Douala, Cameroun. Journal of Applied Biosciences, 37: 2496 - 2507.

Gueye MT, Dogo S, Seynabou B, Kerstin H, Mbacké S, Wathelet J-P, Lognay G. 2011. Insecticidal activity of Boscia senegalensis (Pers.) Lam ex Poir. on Caryedon serratus (OI.) pest of stored groundnuts. African Journal of Agricultural Research, 6(30): 63486353. DOI: 10.5897/AJAR11.949.

Innocent B, Kangpete M, Makalao BI, Ngoubdou G. 2010. Enquête ethnobotanique sur les plantes à effet insecticide et méthodes d'utilisation, à l'échelle traditionnelle, contre les agents vecteurs du paludisme dans la région de l'Extrême-Nord Cameroun: cas de l'arrondissement de Kaélé. Mémoire de Licence, Université de Yaoundé, 55 p.

Issaka H, Badariott D. 2013. Les inondations à Niamey, enjeux autour d'un phénomène complexe. Revue de Géographie de Bordeaux, 263 : 289-392. DOI: 10.4000/com.6900.

Jacques M. 2008. Nouvelles flores illustrées du Sénégal. Famille des Labiées, 28p.

MSP. 1985. Contribution aux études ethnobotaniques et floristiques au Niger, Rapport du Ministère de la Santé Publique du Niger, 250p. 
Denou A, Koudouvo K, Togola A, Haïdara M, Dembele SM, Ballo FN, Sanogo R, Diallo D, Gbeassor M. 2017. Savoir traditionnel sur les plantes antipaludiques à propriétés analgésiques, utilisées dans le district de Bamako (Mali). J. Appl. Biosci., 112 : 10985-10995.

DOI: https://dx.doi.org/10.4314/jab.v112i1.3

Kasali FM, Mahano AO, Kadima NJ, Mpiana PT, Ngbolua KN, Tshibangu TSD. 2014. Ethnopharmacological survey of medicinal plants used against malaria in Butembo City (DR Congo). Journal of Advanced Botany and Zoology, 1(1): $2348-7313$.

Koudouvo K, Karou Ds, Kokou K, Essien Aklikokou Kk, Glitho IA, Simpore J, Sanogo R, Souza CDE, Gbeassor M. 2011. An ethnobotanical study of antimalarial plants in Togo Maritime Region. Journal of Ethnopharmacology, 134: 183-190.

Lawaly MM, Idrissa M, Khalid I. 2017. Les plantes médicinales utilisées dans le traitement des diarrhées au Niger : étude ethnobotanique. Algerian Journal of Natural Products, 4(3): 475-482. DOI: https://doi.org/10.5281/zenodo.1069669.

N'guessan KFH, Tra BI, Kone MW. 2009. Étude ethnopharmacologique des plantes antipaludiques utilisées en médecine traditionnelle chez les Abbey et Krobou d'Agboville

(Côte-d'Ivoire).

Ethnopharmacologia, 44 : 42-50.

Rivière C, Nicolas JP, Caradec ML, Desirea O, Hassan DA, Rémy G, Dupont F. 2005. Importance de l'identification botanique dans la démarche ethnopharmacologique; cas d'une Bignoniaceae malgache, Perichlaena richardii Baill. Acta Botanica Gallica, 152(3): 377-388. DOI: $10.1080 / 12538078.2005 .10515496$
Riviere C, Jean-Pierre N, Marie-Laure C, Odile D, Diny AH, Georges R, Annick D, Frederic D. 2013. Importance de l'identification botanique dans la démarche ethnopharmacologique; cas d'une Bignoniaceae malgache, Perichlaena richardii Baill. Acta Botanica Gallica, 152 (3): 377-388. doi: 10.1080/12538078.2005.10515496.

Karunamoorthi K, Ilango K. 2010. Larvicidal activity of Cymbopogon citratus. European Review for Medical and Pharmacological Sciences, 14 : 57-62.

Khan H, Khan IA, Khan IU, Ohail KS, Khan A, Hussain SH. 2015. The efficacy of some plant extracts against Culex quinquefasciatus (Say) (Diptera: Culicidae). Journal of Entomology and Zoology Studies, 3(3): 212-214.

Saotoing P, Tchuenguem FN, Febal HA, Amba AHB, Amadou H, Nawe TUS. 2014. Evaluation of Insecticidal Effect of Vegetable Oils extracted from the Ripe Seeds of Azadirachta indica and Khaya senegalensis in Adult Anopheles gambiae SL in Maroua, Far North Region Cameroon. Global Journal of Research on Medicinal Plants \& Indigenous Medicine, 3(8): 312-322.

Seye F, Raymond DN, Mady N. 2006. Etude comparative de deux produits de neem (huile et poudre) sur les stades préimaginaux du moustique Culex quinquefasciatus (Diptera : Culicidae). Afrique Science, 02: 212-225.

WHO. 2002. Traditional Medicine Strategy, 2002-2005. Geneva, Switzerland: World Health Organization; Document WHO/EDM/TRM/2002.1. 